|IIIIIIIIIIIIIIIIIIIIIIIIIIIIIIIIIII

Technical Report

IIIIIIIIIIIIIIIIIIIIIIIIIIIIIIIIIIIII

\title{
Effect of sample preparation on the estimation of residue levels of sprayed pesticides in separate analyses of turnip roots and leaves: inclusion or exclusion of the root-shoot junction
}

\author{
Tomonari Yajıma,* Masahiro Fujıta, Kazuaki Iıjıma, Kiyoshi Sato and Yasuhiro Kato \\ The Institute of Environmental Toxicology (IET), 4321, Uchimoriya-machi, Joso-shi, Ibaraki 303-0043, Japan
}

(Received February 28, 2017; Accepted April 10, 2017)

\begin{abstract}
The effect of inclusion or exclusion of the root-shoot junction on the estimation of pesticide residue levels in turnip roots and leaves was investigated. Turnips grown at two experimental sites were sprayed with six pesticides. At residue analysis, the turnips were divided in three segments: roots $(\mathrm{R})$, leaves $(\mathrm{L})$, and root-shoot junctions $(\mathrm{J})$. The highest pesticide residue amounts were found in leaves $\geq 93 \%$ of total, with minimal amounts in roots. Residue amounts in root-shoot junctions were intermediate between those of leaves and roots. Residue levels were calculated for the root plus root-shoot junction, and were higher than those in roots: $(\mathrm{R}+\mathrm{J}) / \mathrm{R}=1.0-9.0$. In contrast, residue levels in the leaf plus root-shoot junction were lower than in leaves only: $(\mathrm{L}+\mathrm{J}) /$ $\mathrm{L}=0.76-0.91$. The results indicate that the position of the cut between root and leaves could greatly affect the estimated pesticide

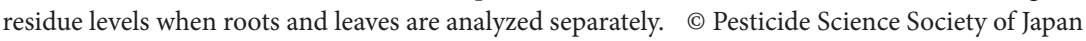

Keywords: analytical portion, leaf, root, turnip, pesticide residue, separate analysis.
\end{abstract}

Electronic supplementary material: The online version of this article contains supplementary material (Supplemental Table S1-S4), which is available at http://www.jstage.jst.go.jp/browse/jpestics/

\section{Introduction}

The methodology of preparing samples of root vegetables for testing pesticide residue levels in roots and leaves is important, as it can affect the test outcomes. As most Japanese people eat both root and leaf portions of turnips and Japanese radishes, it is essential that maximum residue limits (MRLs) are established separately for leaf and root portions of these crops. ${ }^{1)}$ By contrast, the leaves of carrots are not generally eaten, and the MRLs for carrots need only to be established for roots. The FAO manual defines the part of the carrot plant that should be analyzed: "Tops are carefully cut off with a knife by cutting through the bottom of the stem at the lowest point of attachment of the outer petioles. If an annulus of root tissue is thereby severed from hollow-crown roots, the material should be recombined with the roots." ${ }^{2}$ However, no such detailed description is available for turnips and radishes, even though their roots and leaves need to be analyzed separately.

Although the procedure for separating root (R; Fig. 1) and leaf (L; Fig. 1) samples might seem simple, there is a problem with judging how to handle the root-shoot junction (J; Fig. 1).

\footnotetext{
* To whom correspondence should be addressed.

E-mail: yajima@iet.or.jp

Published online June 3, 2017

(c) Pesticide Science Society of Japan
}

The leafy parts of harvested turnips and radishes tend to spoil rapidly, as compared with the root; therefore, roots with only basal portions of leaves are usually sold in the retail market ( $+\mathrm{J}$; Fig. 1). In general, test samples of turnips and Japanese radishes from crop field trials are prepared by separating the leaf and root parts, leaving very small segments of root top with the leaves (L+J; Fig. 1).

Although data on pesticide residues in roots and leaves are available for the turnip and Japanese radish, ${ }^{3-5)}$ there is little information on the effect of the inclusion or exclusion of the rootshoot junction region on residue levels. This study was undertaken to investigate the effects of separate analyses of leaves and roots on determining pesticide residues in turnips. Test samples were obtained from two test fields in Japan. The harvested turnips were divided into roots (R), leaves (L), and root-shoot junctions $(\mathrm{J})$, and the samples were analyzed separately. Pesticide res-
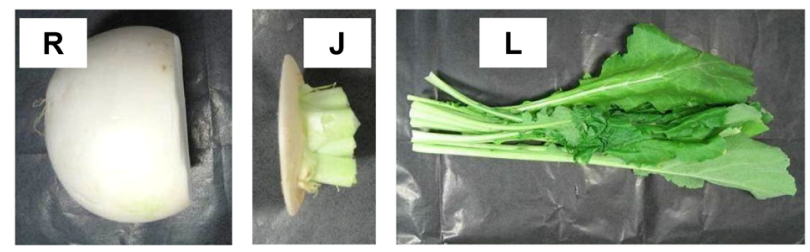

Fig. 1. Separation of a Miyazaki turnip sample into root (R), root-shoot junction $(\mathrm{J})$, and leaf $(\mathrm{L})$ portions. 
idue levels in the root plus junction portions $(\mathrm{R}+\mathrm{J})$ and the leaf plus junction portions $(\mathrm{L}+\mathrm{J})$ were determined to analyze how the sample preparation process affected residue level estimates in turnips. This investigation provides valuable insights into the effect of sample preparation of leaf and root portions on determining the residue levels of sprayed pesticide in turnips.

\section{Materials and Methods}

\section{Field experiments}

Field experiments were conducted in greenhouses (at Ibaraki) and plastic tunnels (at Miyazaki) of the Japan Plant Protection Association in accordance with the Japanese Guidelines for crop field trials. ${ }^{6}$ Turnips of the 'Taibyohikari' cultivar were grown at both sites. Six pesticides were selected to cover a wide range of physicochemical properties: dinotefuran, a neonicotinoid insecticide that has a relatively low $\log P_{\mathrm{OW}}$ of -0.549 and a relatively high water solubility of $39.8 \mathrm{~g} / \mathrm{L} ;^{7)}$ tolfenpyrad, an insecticide that has a relatively high $\log P_{\mathrm{OW}}$ of 5.61 and a relatively low water solubility of $0.087 \mathrm{mg} / \mathrm{L} ;^{7)}$ and acetamiprid, azoxystrobin, chlorfenapyr, and cyazofamid, which are insecticides or fungicides that have intermediate physicochemical properties with respect to $\log P_{\mathrm{OW}}$ and water solubility. These pesticides were applied to turnip fields at the maximum permitted levels, the maximum number of applications, and the minimum pre-harvest intervals. After dilution with water from $1: 3000$ to $1: 1000$, the crops were sprayed one to three times with pesticides, with approximately 4 - or 7-day intervals between each application. The application conditions for each pesticide are summarized in the left columns of Table 1. The pesticides were sprayed with a tank-mix combination of Mospilan ${ }^{\circledR}$ water soluble powder of acetamiprid $20.0 \%$ a.i. (Nippon Soda Co., Ltd.), Amistar ${ }^{\circledR} 20$ flowable of azoxystrobin $20.0 \%$ a.i. (Syngenta Japan K.K.), Kotetsu $^{\circledR}$ flowable of chlorfenapyr $10.0 \%$ a.i. (Nippon Soda Co., Ltd.), Ranman ${ }^{\circledR}$ flowable of cyazofamid $10.0 \%$ a.i. (ISK Biosciences K.K.), Starkle ${ }^{\circledR}$ water-dispersible granules of dinotefuran $20.0 \%$ a.i. (Mitsui Chemicals Agro, Inc.), and Hachi-Hachi ${ }^{\circledR}$ emulsifiable concentrate of tolfenpyrad $15.0 \%$ a.i. (OAT Agrio Co., Ltd.) using back-carried sprayers with corn nozzles. Application rates ranged from 183 to $186 \mathrm{~L} / 10 \mathrm{a}$. The final applications of Mospilan, Amistar, Kotetsu, Ranman, Starkle, and HachiHachi were 21, 7, 1, 3, 3, and 7 days before harvest, respectively. More than $5 \mathrm{~kg}$ of turnips (25 turnips in Ibaraki, 5 turnips in Miyazaki) was obtained at random from the test fields. Intact turnips, without separation of leaves and roots, were shipped at $3^{\circ} \mathrm{C}$ to our institute by a commercial shipping service.

\section{Sample preparation}

Each turnip was weighed, and then divided into root, leaf, and root-shoot junction parts. The mean weights of whole turnips of $269 \mathrm{~g}$ ( $6.72 \mathrm{~kg} / 25$ turnips) and $1.10 \mathrm{~kg}$ (5.50 kg/5 turnips) were obtained for the Ibaraki and Miyazaki samples, respectively. The root-shoot junction was defined as the region $1-1.5 \mathrm{~cm}$ from the attachment point of the leaves to the root (see Fig. 1). The weight ratios of root-to-junction-to-leaf were 56:6:39 for the Ibaraki sample and 56:11:33 for the Miyazaki sample. The samples were kept at $-20^{\circ} \mathrm{C}$ until analysis. Frozen samples were homogenized using a 3901JP blender (Russell Hobbs) before analysis.

Table 1. Pesticide residue levels in the root, root-shoot junction and leaf portions of turnips

\begin{tabular}{|c|c|c|c|c|c|c|}
\hline \multirow[b]{2}{*}{ Pesticide, application ${ }^{a)}$} & \multirow[b]{2}{*}{ Field location } & \multicolumn{3}{|c|}{ Measured residue levels ${ }^{b)}$} & \multicolumn{2}{|c|}{ Calculated residue levels ${ }^{c)}$} \\
\hline & & $\begin{array}{l}\text { root } \\
(\mathrm{R})\end{array}$ & $\begin{array}{l}\text { junction } \\
(\mathrm{J})\end{array}$ & $\begin{array}{l}\text { leaf } \\
(\mathrm{L})\end{array}$ & $\begin{array}{l}\text { root with junction } \\
\qquad(\mathrm{R}+\mathrm{J})\end{array}$ & $\begin{array}{l}\text { leaf with junction } \\
\qquad(\mathrm{L}+\mathrm{J})\end{array}$ \\
\hline Acetamiprid & Ibaraki & $0.01(1.8 \%)$ & $0.02(0.4 \%)$ & $0.78(98 \%)$ & $0.01(1.0)$ & $0.69(0.88)$ \\
\hline 2000 times $\times 1$ ( 21 day) & Miyazaki & $<0.01$ & $0.01(0.4 \%)$ & $0.94(99 \%)$ & 0.01 & $0.71(0.76)$ \\
\hline Azoxystrobin & Ibaraki & $0.02(0.5 \%)$ & $1.29(3.0 \%)$ & $5.88(97 \%)$ & $0.13(6.5)$ & $5.31(0.90)$ \\
\hline 2000 times $\times 2$ ( 7 day) & Miyazaki & $<0.01$ & $0.34(1.9 \%)$ & $5.86(98 \%)$ & 0.06 & $4.47(0.76)$ \\
\hline Chlorfenapyr & Ibaraki & $0.02(0.6 \%)$ & $1.04(2.9 \%)$ & $4.99(97 \%)$ & $0.11(5.5)$ & $4.50(0.90)$ \\
\hline 2000 times $\times 2$ (1 day) & Miyazaki & $<0.01$ & $0.22(1.9 \%)$ & $3.84(98 \%)$ & 0.04 & $2.93(0.76)$ \\
\hline Cyazofamid & Ibaraki & $0.01(0.3 \%)$ & $0.87(2.8 \%)$ & $4.26(97 \%)$ & $0.09(9.0)$ & $3.84(0.90)$ \\
\hline 2000 times $\times 3$ (3 day) & Miyazaki & $<0.01$ & $0.34(2.6 \%)$ & $4.24(97 \%)$ & 0.06 & $3.26(0.77)$ \\
\hline Dinotefuran & Ibaraki & $0.07(3.5 \%)$ & $0.74(3.6 \%)$ & $2.70(93 \%)$ & $0.13(1.9)$ & $2.46(0.91)$ \\
\hline 3000 times $\times 2$ ( 3 day) & Miyazaki & $0.02(0.7 \%)$ & $0.40(2.9 \%)$ & $4.46(96 \%)$ & $0.08(4.0)$ & $3.44(0.77)$ \\
\hline Tolfenpyrad & Ibaraki & $0.06(1.5 \%)$ & $1.57(3.9 \%)$ & $5.36(95 \%)$ & $0.20(3.3)$ & $4.89(0.91)$ \\
\hline 1000 times $\times 2$ ( 7 day) & Miyazaki & $<0.01$ & $0.58(2.9 \%)$ & $6.54(97 \%)$ & 0.10 & $5.04(0.77)$ \\
\hline
\end{tabular}

a) Dilution factors of each formulation $\times$ number of application (Pre-harvest interval).

${ }^{b)}$ Mean of the measured residue levels in root $(\mathrm{R})$, root-shoot junction $(\mathrm{J})$ and leaf $(\mathrm{L})$ portions of turnips $(n=2, \mathrm{mg} / \mathrm{kg})$. The value in the parentheses represents percentage distribution to the whole crop.

c) Calculated residue levels in the root and leaf portions with the junction portion. A half-value of the limit of quantification $(0.005 \mathrm{mg} / \mathrm{kg}) \mathrm{was}$ used for the calculations, where the residue level was $<0.01 \mathrm{mg} / \mathrm{kg}$. The value in the parentheses represents ratio of the calculated residue level to the measured residue level. 


\section{Residue analysis}

Residue analytical methods were optimized to allow the rapid analysis of each pesticide in the various portions of turnip, as described below.

\subsection{Chemicals and reagents}

Analytical standards (purity $\geq 98.9 \%$ ) of acetamiprid, azoxystrobin, chlorfenapyr, cyazofamid, dinotefuran, and tolfenpyrad were purchased from Hayashi Pure Chemical and Wako Pure Chemical Industries. Pesticide analysis-grade acetonitrile and toluene, LC-MS-grade acetonitrile, and analytical-grade ammonium acetate were purchased from Wako Pure Chemical Industries. Water used for the experiments was purified using a MilliQ system (Millipore).

Standard stock solutions $(200 \mathrm{mg} / \mathrm{L})$ of each pesticide were separately prepared with acetonitrile. Aliquots of each stock solution were mixed and diluted with an acetonitrile-water solution to make standard solutions in the range of $0.1-4 \mu \mathrm{g} / \mathrm{L}$ to prepare calibration curves.

\subsection{Extraction}

Twenty grams of homogenized sample was placed in an Erlenmeyer flask and extracted with $100 \mathrm{~mL}$ of acetonitrile by shaking for $30 \mathrm{~min}$ using a reciprocal shaker. The mixture was filtered by vacuum suction, and the residual sediment was washed with $50 \mathrm{~mL}$ of acetonitrile and then filtered again. The filtrates were combined and made up to a volume of $200 \mathrm{~mL}$ with acetonitrile.

\subsection{Cleanup}

A $1-\mathrm{mL}$ aliquot $(0.1 \mathrm{~g}$ of the sample) of the acetonitrile extract was diluted with $10 \mathrm{~mL}$ of water, and the mixture was loaded onto a graphite carbon black cartridge $(500 \mathrm{mg} / 6 \mathrm{~mL}$, Supelclean ENVI-Carb; Sigma-Aldrich, conditioned with acetonitrile and water before use) for solid-phase extraction. The cartridge was washed with $10 \mathrm{~mL}$ of acetonitrile-water mixture $(1: 9, \mathrm{v} / \mathrm{v})$. After aspirating the cartridge for $1 \mathrm{~min}, 5 \mathrm{~mL}$ of acetonitrile and $10 \mathrm{~mL}$ of acetonitrile-toluene mixture $(3: 1, \mathrm{v} / \mathrm{v})$ were passed through the cartridge sequentially, and the eluate was collected. The eluate was evaporated in a $40^{\circ} \mathrm{C}$ water bath, and the residue was dried in a gentle stream of nitrogen. The residue was dissolved in a suitable volume $(5-500 \mathrm{~mL})$ of an acetonitrile-water mixture $(1: 1, \mathrm{v} / \mathrm{v})$, and then the test solution was injected into the LC-MS/MS system.

\subsection{LC-MS/MS analysis}

The level of each pesticide in the injected solution was determined by a linear regression analysis of each standard calibration curve and by comparing the peak area to each concentration in the sample. An LC-MS/MS (Model 1290 Infinity Pumping System; MS/MS, Model 6460 Triple Quadrupole Tandem Mass Spectrometer; Agilent Technologies) equipped with an electrospray interface operating in positive (for acetamiprid, azoxystrobin, cyazofamid, dinotefuran, and tolfenpyrad) or negative (for chlorfenapyr) ion mode was used. The data were processed using Agilent MassHunter (version B03.01). LC separation was performed using a ZORBAX Eclipse Plus C18 column $(2.1 \mathrm{~mm} \times 50 \mathrm{~mm}, 1.8 \mu \mathrm{m}$; Agilent Technologies $)$ at $40^{\circ} \mathrm{C}$.

Acetonitrile and $5 \mathrm{mmol} / \mathrm{L}$ ammonium acetate aqueous solu- tion were used as the mobile phase at a flow rate of $0.4 \mathrm{~mL} / \mathrm{min}$. For the gradient-elution analysis, the ratios of the acetonitrile and aqueous solution were as follows: 5:95(for $2 \mathrm{~min}$ ) $-2 \mathrm{~min}-$ $60: 40-3$ min-90:10. The MS parameters were as follows: capillary voltage, $2500 \mathrm{~V}$; nebulizer gas, $35 \mathrm{psi}$; drying gas, $5 \mathrm{~L} / \mathrm{min}$ $\left(300^{\circ} \mathrm{C}\right)$; and sheath gas, $10 \mathrm{~L} / \mathrm{min}\left(400^{\circ} \mathrm{C}\right)$.

For chlorfenapyr determination, a pump was set under the same conditions described above except for the gradient program. The ratio of the acetonitrile and aqueous solution was as follows: $50: 50$ (for $1 \mathrm{~min}$ ) $-3 \mathrm{~min}-80: 20$. The MS parameters were as follows: capillary voltage, $-2000 \mathrm{~V}$; nebulizer gas, $45 \mathrm{psi}$; drying gas, $8 \mathrm{~L} / \mathrm{min}\left(200^{\circ} \mathrm{C}\right)$; and sheath gas, $12 \mathrm{~L} / \mathrm{min}\left(400^{\circ} \mathrm{C}\right)$.

The MS detection was set to multiple-reaction monitoring mode. The injection volume was set at $10 \mu \mathrm{L}$. The retention times, fragmentor voltages, collision energies, and monitoring ions are summarized in Supplemental Table S1. The five pesticides except chlorfenapyr obtained $[\mathrm{M}+\mathrm{H}]^{+}$ion as a precursor ion at satisfactory sensitivity. The precursor ion of chlorfenapyr was employed $\left[\mathrm{M}-\mathrm{CH}_{2} \mathrm{OC}_{2} \mathrm{H}_{5}\right]^{-}$ion, because of the difficulty of detecting protonated or deprotonated ion on our optimizing process.

\section{Validity of the analytical method}

Results of the recovery and stability tests for the roots, leaves, and root-shoot junctions of turnips are summarized in Supplemental Tables S2, S3, and S4, respectively. The accuracy and precision of the analytical methods were evaluated by recovery tests on pesticides at two or more spiked levels, including a level at a limit of quantification of $0.01 \mathrm{mg} / \mathrm{kg}$ and above the highest residue levels. The mean recoveries of spiked samples across a six-fold concentration ranged from 87 to $105 \%$ with relative standard deviations of $\leq 8.1 \%$. The specificity of the analytical method was evaluated by analyzing duplicate blank samples, which were obtained from both test sites. No interference peak was observed around the retention time of any pesticide on the chromatograms from the blank samples.

Twenty grams of homogenized sample was used for the stability tests, which were conducted at a concentration level of $0.1 \mathrm{mg} / \mathrm{kg}$ (samples stored at $-20^{\circ} \mathrm{C}$ for 131 or 135 days). Recoveries from the stability samples $(n=36)$ were within an acceptable range (70-120\%).

From these results, the analytical methods used in this study were confirmed to generate robust data for determining pesticide residues in separate analyses of roots, leaves, and root-shoot junctions in turnips.

\section{Statistical analysis}

Wilcoxon signed rank test was conducted using $R$ (ver. 3.3.2) for Windows, which was obtained from the website of the Comprehensive $R$ Archive Network (http://cran.r-project.org/).

\section{Results and Discussion}

\section{Measured residue data}

The results of the analyses of residue levels in roots, leaves, and 
root-shoot junctions are summarized in Table 1. Pesticide residue levels in roots ranged from $<0.01-0.07 \mathrm{mg} / \mathrm{kg}$ (dinotefuran, Ibaraki). Residue levels in roots from the Miyazaki field were lower than those from Ibaraki. The residue levels of five pesticides were lower than the limits of quantification in roots from Miyazaki; the exception was dinotefuran. On the other hand, all pesticides were detected in roots from the Ibaraki field. This difference in residue levels may be due to differences in the weights of the turnips between the two test sites, and the difference in mean sample weights between the Ibaraki and Miyazaki fields may have resulted from several factors, such as differences in soil texture (Ibaraki, loam; Miyazaki, sandy loam) or weather conditions. In contrast, the turnip leaves showed considerably higher levels of pesticide residues. These ranged from $0.78 \mathrm{mg}$ / $\mathrm{kg}$ (acetamiprid, Ibaraki) to $6.54 \mathrm{mg} / \mathrm{kg}$ (tolfenpyrad, Miyazaki). Pesticide residue levels in the root-shoot junctions of turnips ranged from $0.01 \mathrm{mg} / \mathrm{kg}$ (acetamiprid, Miyazaki) to $1.57 \mathrm{mg}$ / $\mathrm{kg}$ (tolfenpyrad, Ibaraki). The levels were intermediate between those found in roots and leaves. The levels of pesticide residues determined in this study were lower than the Japanese MRLs specified by the Japanese Food Sanitation $\mathrm{Law}^{\mathrm{l})}$ for turnip roots (acetamiprid, $0.1 \mathrm{mg} / \mathrm{kg}$; azoxystrobin, $1 \mathrm{mg} / \mathrm{kg}$; chlorfenapyr, $0.2 \mathrm{mg} / \mathrm{kg}$; cyazofamid, $0.3 \mathrm{mg} / \mathrm{kg}$; dinotefuran, $0.5 \mathrm{mg} / \mathrm{kg}$; and tolfenpyrad, $1 \mathrm{mg} / \mathrm{kg}$ ) and for turnip leaves (acetamiprid, $5 \mathrm{mg} /$ $\mathrm{kg}$; azoxystrobin, $15 \mathrm{mg} / \mathrm{kg}$; chlorfenapyr, $15 \mathrm{mg} / \mathrm{kg}$; cyazofamid, $20 \mathrm{mg} / \mathrm{kg}$; dinotefuran, $5 \mathrm{mg} / \mathrm{kg}$; and tolfenpyrad, $25 \mathrm{mg} / \mathrm{kg}$ ).

In considering the proportion of total plant pesticide residues, the roots were estimated to contain up to $3.5 \%$, the leaf portion had more than $93 \%$, and the root-shoot junctions contained $\leq 3.9 \%$. No significant differences were observed in the distribution patterns of pesticide residues among the three portion samples, despite these being prepared from turnips grown at two separate locations and being treated with six pesticides with different physicochemical properties (Fig. 2). From the results described in this section, the pre-harvested residue data sets obtained are considered to adequately provide representa-

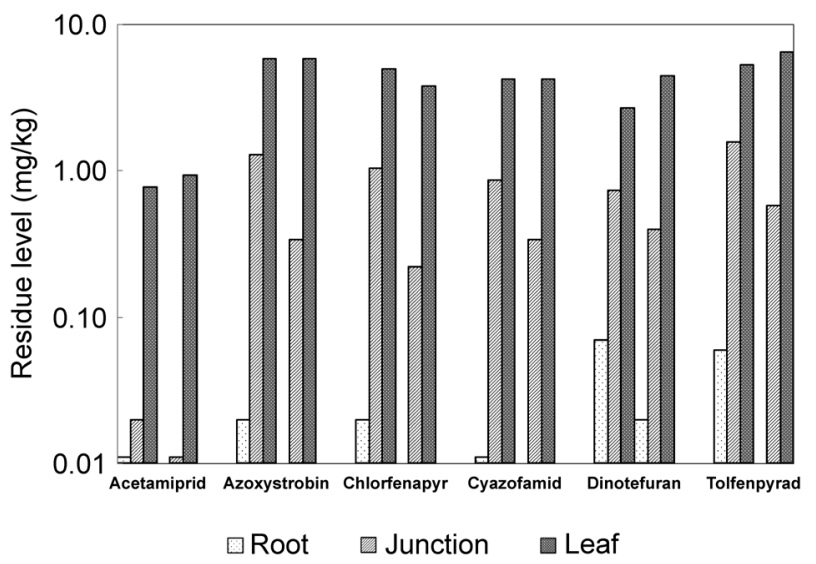

Fig. 2. Residue levels of six pesticides in the root, leaves, and root-shoot junctions of turnips sampled from Ibaraki (right side of each pesticide) and Miyazaki (left side of each pesticide).

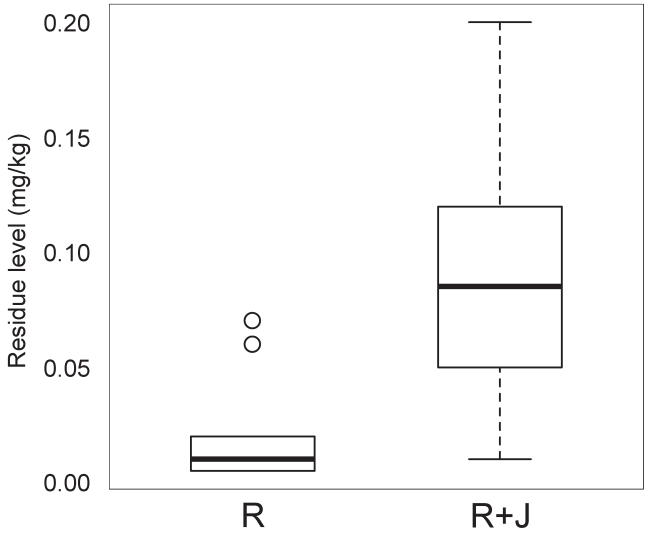

Fig. 3. Box plots of variability in the measured residue levels of six pesticides in the root portions $(\mathrm{R})$ and the calculated residue levels in the root portion with root-shoot junctions $(\mathrm{R}+\mathrm{J})$. The bottom and top of the box indicate the lower and the upper quartile points, respectively, and the lines from the bottom to the top indicate the range from the minimum to the maximum residue levels of pesticides.

tive pesticide residue data for turnips, according to normal Japanese agricultural practices.

\section{Handling effect of the root-shoot junction}

The pesticide residue levels in roots and leaves were recalculated after including root-shoot junction data (Table 1, right-hand columns). For this recalculation, we applied the rule that a half value of the limit of quantification $(0.005 \mathrm{mg} / \mathrm{kg})$ should be used when the residue level was $<0.01 \mathrm{mg} / \mathrm{kg}$.

The calculated pesticide residue levels in roots plus root-shoot junctions were higher than those in roots alone. Pesticide residue levels in the roots plus root-shoot junctions were all calculated to be detectable values, even though the measured values of roots only were non-detectable for the Miyazaki samples. The

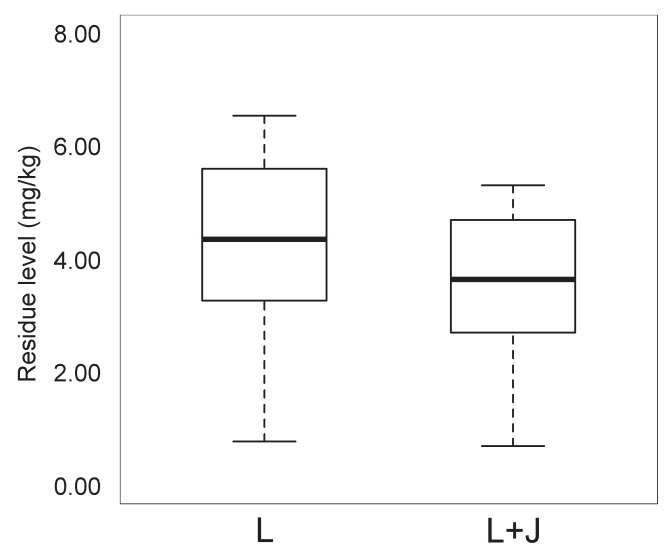

Fig. 4. Box plots of variability in the measured residue levels of six pesticides in the leaf portions (L), and the calculated residue levels in the leaf portion with root-shoot junctions $(\mathrm{L}+\mathrm{J})$. Bottom and top of the box indicate the lower and the upper quartile points, respectively, and the lines from bottom to top indicate the range from the minimum to the maximum residue levels of pesticides. 
largest difference in residue levels between roots and roots plus root-shoot junctions was observed for cyazofamid in turnips grown at Ibaraki.

In contrast, the calculated pesticide residue levels for leaves plus root-shoot junctions were lower than those for leaves only. The ratios of residue levels in leaves plus root-shoot junctions to leaves only $((\mathrm{L}+\mathrm{J}) / \mathrm{L})$ ranged from $0.88-0.91$ in the Ibaraki field sample, and from $0.76-0.77$ in the Miyazaki field sample. The ratios in the latter were likely lower because the percentage weight of the root-shoot junction to the whole crop was greater in the Miyazaki sample (11\%) than in the Ibaraki sample (6\%), which might have caused the dilution effect.

Boxplots of residue levels in roots and leaves and their combinations with the root-shoot junctions are shown in Fig. 3 and 4 , respectively. Statistical analysis using the Wilcoxon signed rank test indicated that there were significant differences between samples with root-shoot junctions as compared to samples without root-shoot junctions (roots, $p=0.0009766$; leaves, $p=0.0004884$ ). The variabilities in residue levels of the six pesticides in root samples were obviously wider than those of leaves with and without root-shoot junctions.

\section{Conclusion}

Based on the limited results of this study, the sample preparation process was found to have a significant effect on pesticide residue levels when analyzing turnip roots and leaves separately, depending on whether the samples included root-shoot junctions. The selection of the position of cutting to separate the root and leaf portions had a more severe effect on pesticide residue levels in the root portions than in the leaves because the residue levels in roots were lower than those in leaves after the foliar applica- tion of pesticides. These results suggest that care must be taken in the separation process to obtain accurate analytical results for pesticide residue analyses of turnip roots and leaves.

\section{Acknowledgements}

We thank the staff at the Japan Plant Protection Association for their cooperation in the field experiments. This research was performed as part of the "Shokuhin-no-Anshin-Anzenkakuho-Suishin-Jigyou," sponsored by the Japanese Ministry of Health, Labour and Welfare.

\section{References}

1) The Japan Food Chemical Research Foundation: "Maximum Residue Limits (MRLs) List of Agricultural Chemicals in Foods" http://www. m5.ws001.squarestart.ne.jp/foundation/search.html (accessed January 27, 2017).

2) Food and Agriculture Organization of the United Nations: "FAO Plant Production and Protection Paper 197, Submission and Evaluation of Pesticide Residues Data for the Estimation of Maximum Residue levels in Food and Feed," Rome, Italy, 2009.

3) G. A. Donald: J. Assoc. Off. Anal. Chem. 63, 1109-1113 (1980).

4) G. A. Donald: J. Assoc. Off. Anal. Chem. 68, 1160-1163 (1985).

5) R. Saito, S. Ishihara, T. Yokoyama, K. Someya, A. Fujimoto, T. Izumisawa, S. Takamine, Y. Kudo, M. Takanashi, Y. Kitamura and H. Kameda: Research Report of Agricultural Chemicals. 3, 8-13, Food and Agricultural Materials Inspection Center, (2011), in Japanese.

6) Agricultural Production Bureau, Ministry of Agriculture, Forestry and Fisheries of Japan. The guidelines related to the study reports for the registration application of pesticide. Appendix to Director General Notification No. 12-Nousan-8147. Nov. 24, 2000 (Final amendments were made on April 1, 2011)

7) C. D. S. Tomlin (eds.): “The e-Pesticide Manual version 5.0.1," British Crop Protection Council, Hampshire, U.K., 2010. 